\title{
Optical Fiber Sensors for Point Investigation of Cancer Tissues
}

\author{
E. Beres-Pawlik ${ }^{a, *}$, K. Gasiorek ${ }^{a}$, Z. Kulas $^{a}$, M. RzacA $^{b}$ And R. Czarnecki ${ }^{b}$ \\ ${ }^{a}$ Institute of Telecommunications, Teleinformatics and Acoustics, Wrocław University of Technology \\ Wybrzeże Wyspiańskiego 27, 50-370 Wrocław, Poland \\ ${ }^{b}$ Provincial Specialist Hospital, Kamińskiego 73a, 51-124 Wrocław, Poland
}

\begin{abstract}
The paper presents optical fiber sensors for point investigation of cancer tissues. The construction of several sensors has been described, including selection of proper optical fiber as well as the construction of measuring head. Being part of medical endoscopes, presented sensors can be applied to investigate autofluorescence spectra in various tissue areas. The level of autofluorescence intensity indicates the stage of disease advancement. The paper presents the results of investigating autofluorescence spectra for different cancer tissues. Various areas of cancer tissues have been investigated in order to determine the level of disease advancement. In the paper new constructions of fluorescent fiber sensors are presented. The sensors were used to investigate human and animal normal and tumor tissues. The fiber sensors, possible to use in videoendoscopy, are very small. The comparative analysis of fluorescent spectra of normal and tumor tissues is presented.
\end{abstract}

PACS numbers: 47.63.Cb, 87.19.U-, 87.19.Hr

\section{Introduction}

The phenomenon of tissue fluorescence has been used for many years in the diagnosis of various diseases. Areas in which to examine the fluorescence spectra are subjectively selected by the researcher. Evaluation of spectra obtained is relevant to assist in decision making regarding further treatment of the disease.

Worldwide, there are many well-known constructions of the measurement systems which investigate tissue fluorescence. Among them is fluorescence microscopy where the application of appropriate band-pass filters allows the analysis of fluorescent images obtained by exposing organic matter to appropriate wavelengths. Spectrofluorometry of natural fluorophores extracted from the tissue is investigated in order to determine their contribution to the intensity of fluorescence illumination $[1,2]$. There are measuring systems for recording fluorescence images by means of endoscopic systems and CCD cameras [3-5]. Such images are subject to treatment and analysis of digital system by using a special numerical procedures. In some cases, existing endoscopic systems are additionally equipped with fluorescence sensors [6-8]. Endoscope is then fitted with an appropriate miniature optical set used as a measuring head and as the optical fiber transmission path, leading the signal to the apparatus which analyzes the spectrum of fluorescent light. There are also different complex optical systems for diagnosing tissue fluorescence outside the living organism $[9,10]$.

* corresponding author; e-mail: elzbieta.pawlik@pwr.wroc.pl
The basic elements of each sensor measuring system are the measuring heads and transmission paths. Optical fiber sensors are most appropriate for application in this field, due to their small size, sensitivity and easy transmission of optical information.

In this paper we present designed and constructed fiber sensors and the preliminary measurements of autofluorescence illumination spectra obtained by their means. The presented sensors can be used to work independently, as well as constitute an additional part of endoscopic systems. Due to their small sizes, the sensors allow for point investigation of cancer tissues.

\section{The construction of optical fiber sensors}

A step-index fiber of sol-gel glass manufactured at the Laboratory of Fiber Technology in Lublin was used for the construction of optical fiber sensors. The same type of fiber was used for optical fibers which are responsible for providing UV radiation to the tissue, as well as for collecting fluorescence response. The fiber has a core diameter of $200 \mu \mathrm{m}$, coat diameter of $260 \mu \mathrm{m}$ and protective cover diameter of $340 \mu \mathrm{m}$.

Two kinds of measuring head constructions were used. Tissue illumination in the sensors was always performed by means of a single fiber so that information on the autofluorescence spectrum of the investigated tissue would be collected from possibly small area. Either one or six optical fibers were used to collect feedback information (autofluorescence illumination spectrum) from the tissue, depending on the needs. The transmission path length was $2 \mathrm{~m}$. The fiber ends were carefully polished 


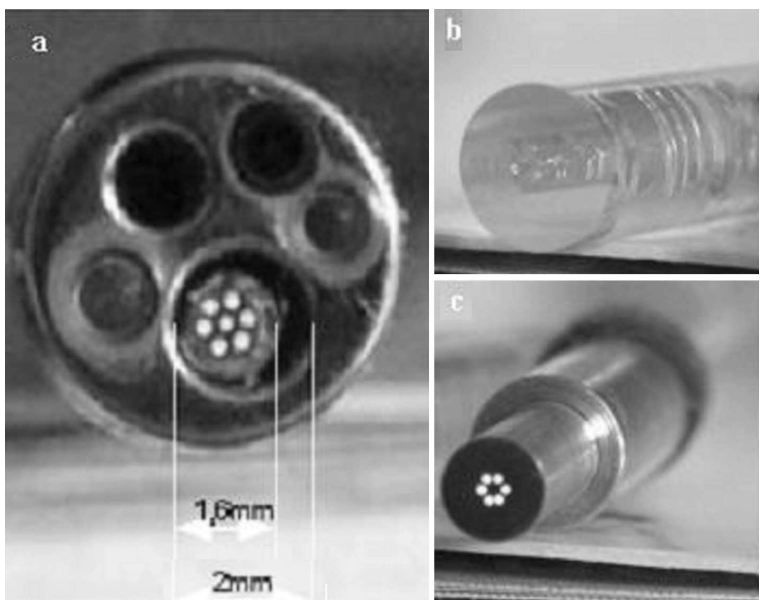

Fig. 1. Seven-fiber sensor: (a) sensor head placed in bronchoscope, (b) element introducing exciting light to light-emitting fiber, (c) connector between light-receiving fibers and measuring apparatus.

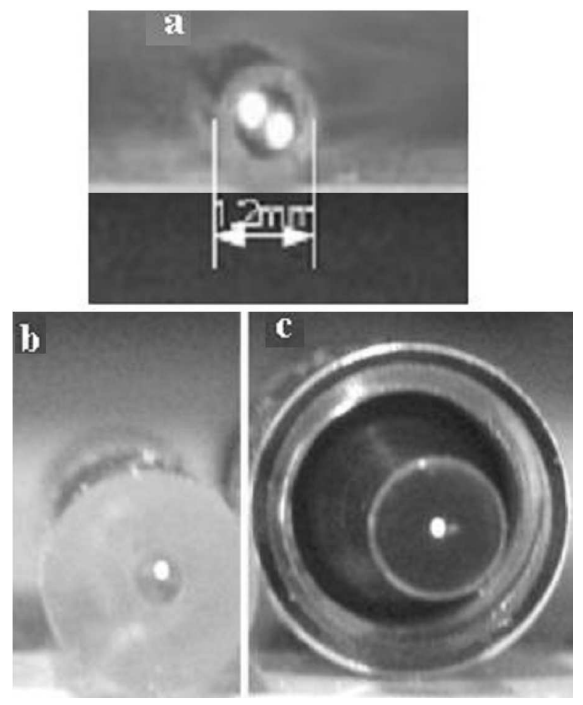

Fig. 2. Two-fiber sensor: (a) the sensor head, (b) element introducing exciting light to light-emitting fiber, (c) connector between receiving fibers and measuring apparatus.
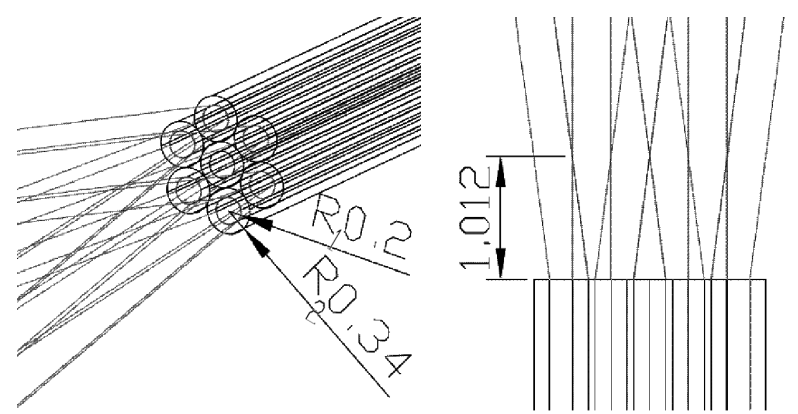

Fig. 3. Light beams propagation in the proposed seven-fiber optical sensor. $\mathrm{R}_{1}$ - fiber core diameter $[\mathrm{mm}], \mathrm{R}_{2}$ - outer diameter of the fiber $[\mathrm{mm}]$. perpendicularly to the core axis and provided with handles to fit the sensors in endoscopic systems. Initial adaptation of sensors was carried out to achieve compatibility with Olympus videobronchoscope type 2T10.

Two constructed optical sensors are shown below. They consist of a light-emitting optical fiber which excites the tissue with UV light causing autofluorescence, and receiving fibers which transmit fluorescent light spectra. The ends of sensor heads are shown below: seven-fiber and two-fiber, Fig. 1 and Fig. 2. Figure 1a presents the dimensions of the measuring heads and an endoscopic hole where these heads were fixed.

The centric fiber was used as the light-emitting fiber in the head of the seven-fiber optical sensor, whilst the other six surrounding fibers were the light-receiving fibers. The two-fiber sensor consists of one light-emitting and one light-receiving fiber.

Light acceptance angles of the adjacent fibers cross each other at the distance of $1.01 \mathrm{~mm}$ from the measuring head, which results from the geometry of the projected systems (Fig. 3). For these constructed optical sensors it is the distance for which the sensor has the highest sensitivity.

\section{Measuring results}

The constructed optical sensors were tested in order to determine optimal distance for the investigated tissue. Fluorescence intensity was measured as a function of sensor's distance from the investigated specimen. The characteristics thus obtained determine the distance between measuring head and tissue. In case the measurement has been carried out for wrong distance value, the results of fluorescence intensity can be corrected against the curves shown below. However, it would be advisable to perform the measurements only for one maximum fluorescence intensity (Fig. 4).

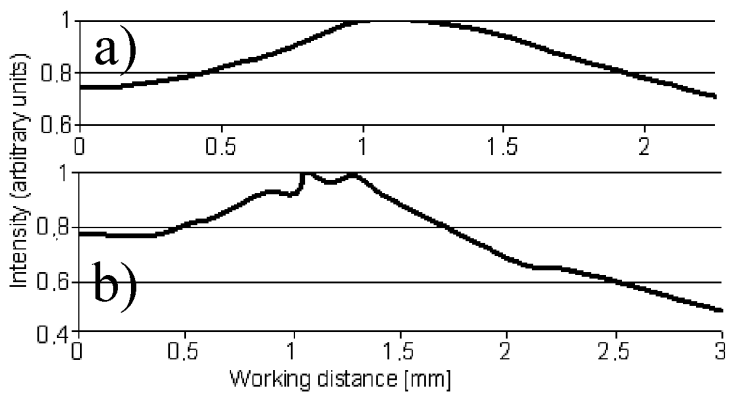

Fig. 4. Measurement of maximum fluorescence intensity as a function of distance from the tissue: (a) seven-fiber sensor, (b) two-fiber sensor.

Maximum sensitivity distances for seven-fiber sensor were $1.18 \mathrm{~mm}$, and $1.06 \mathrm{~mm}$ for two-fiber sensor, which is consistent with the estimated $1.01 \mathrm{~mm}$ result of maximum fluorescence intensity. The distance of $1 \mathrm{~mm}$ was determined as an appropriate measuring distance. 
The seven-fiber sensor gives about 10 times greater light fluorescence intensity as compared with the two-fiber sensor due to the number of fibers used. It allows recording higher fluorescence intensity, thus enabling easier comparison of the results of point-investigation of the tissues. However, it must be remembered that fluorescence response is collected from a larger surface area. One of these proposed sensors can be used, depending on the type of tissue, its age, or the characteristics of individual patients.

The experiment was performed at the Provincial Specialist Hospital in Wrocław.

A comparison of tissue fluorescence light spectrum of temporal lobe in healthy and diseased tissue areas is shown in Fig. 6b. Presented below are exemplary measurements of tissue autofluorescence intensity performed by means of a two-fiber sensor in accordance with the model shown in Fig. 5. Measuring points are given in the photograph (Fig. 6a).

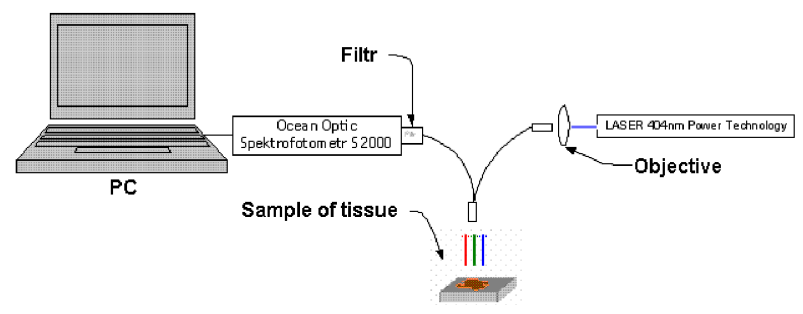

Fig. 5. Measuring system for fluorescence light spectrum.

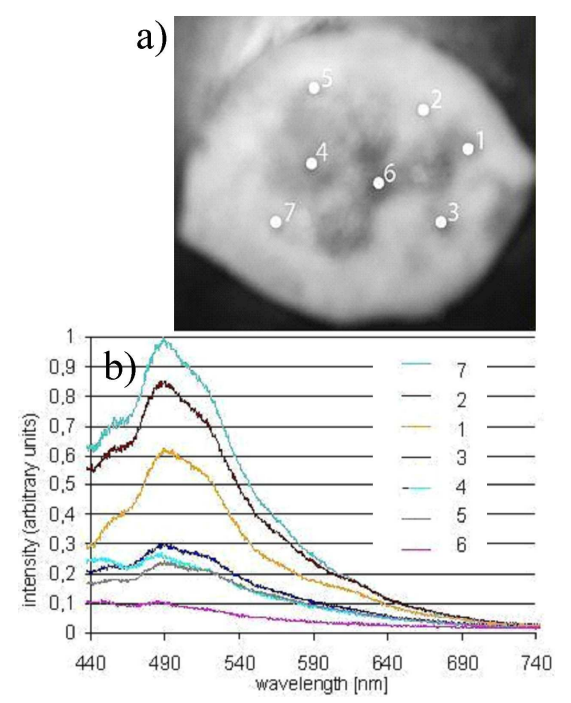

Fig. 6. Investigation of autofluorescence of temporal lobe malignant melanoma.

The experiment was performed in a darkroom. The tissue was supplied with $7.6 \mathrm{~mW}$ optical power (UV range) passing through the fiber, from a semiconductor laser source of $404 \mathrm{~nm}$ wavelength. The beam delivered to the tissue is a divergent beam, according to the fiber acceptance angle. The distance from sensor to tissue was $1 \mathrm{~mm}$.

The results of this investigation are consistent with the visual diagnosis of melanoma. The measurements of the unstained spots give a high autofluorescence intensity. It means that the tissue is healthy. Measurements in section 6 , which is the most inflamed area, show the lowest intensity of fluorescence light. Other measurements of lesioned tissue have given intermediate autofluorescencence intensities. The results of measurements were compared with histopathological examination results. The compatibility of both tests was high.

It should be noted that, while these measurements were performed, only slight autofluorescence was observed coming from the optical fibers used in the test. It could have a minor impact on the measurement results. The fluorescence investigation of optical fibers used in the construction of sensors was carried out in order to select proper fibers to their respective structures.

\section{Summary}

The paper presented designing, construction and testing of optical sensors using sol-gel fibers to perform a non-invasive optical biopsy. The constructed sensors were applied to videoendoscopic medical equipment in use. The preliminary results of the autofluorescence intensity of cancer tissues were presented.

\section{References}

[1] A. Perchant, in: Biomedical Imaging: Nano to Macro, Vol. 1, IEEE, New York 2004, p. 692.

[2] A. Palasz, A. Grobelny, E. Beres-Pawlik, M. Fraczek, M. Zalesska-Krecicka, A. Klimczak, T. Kręcicki, Auris Nasus Larynx 30, 385 (2003).

[3] Z. Kulas, E. Beres-Pawlik, T. Krecicki, J. Non-Cryst. Solids 352, 5055 (2006).

[4] B. Mayinger, Gastrointestinal Endoscopy 59, (2004).

[5] K.T. Schomacker, Gastroenterology 102, (1992).

[6] F. Fisher, Lasers Medical Sci. 16, 9834 (2001).

[7] P. Gibinski, E. Maciak, Z. Opilski, T. Pustelny, A. Sieron, M. Urbanczyk, Mol. Quant. Acoust. 28, 217 (2007).

[8] M. Gawlikowski, T. Pustelny, B. Przywara-Chowaniec, P. Struk, Acta Phys. Pol. A 114, A-79 (2008).

[9] E. Beres-Pawlik, F. Dybala, W. Michalski, D. Dus, Opt. Appl. 34, 385 (2004).

[10] H.W. Wang, IEEE Trans. Biomed Eng. 10, 1273 (1999). 